\title{
Electrospun nanofibers fabricated on screen-printed PZT cantilevers for room temperature VOCs detection
}

\author{
C. R. Zamarreño ${ }^{1}$, F. J. Arregui ${ }^{1}$, Y. R. García ${ }^{1}$, R. Vázquez ${ }^{2}$, E. Llobet ${ }^{2}$, R. Lakhmi ${ }^{3}$, P. Clement ${ }^{3}, H$. \\ Debéda ${ }^{3}$ \\ ${ }^{1}$ Public University of Navarra, Campus Arrosadia, 31006 Pamplona SPAIN \\ ${ }^{2}$ University Rovira I Virgili, 26 Av. Països Catalans, 43007 Tarragona SPAIN \\ ${ }^{3}$ Université de Bordeaux, Laboratoire IMS, 33405 Talence Cedex FRANCE \\ carlos.ruiz@unavarra.es
}

\begin{abstract}
:
The present work describes the utilization of screen-printed PZT cantilevers for the detection of volatile organic compounds (VOCs) at room temperature by means of a specific sensitive coating. The sensitive coating is formed by polyvinylidene fluoride (PVDF) and active carbon electrospun nanofibers with an average diameter of $500 \mathrm{~nm}$. These fibers enable to maximize the surface interaction area and hence the diffusion processes of the molecules within the sensitive coating. When the sensitive coating is exposed to different VOCs and concentrations, the absorption and desorption processes induce shifts in the cantilever resonance frequency value. In particular, a shift of $971 \mathrm{~Hz}$ is observed at a frequency around $802 \mathrm{KHz}$ when the device is exposed to $750 \mathrm{ppm}$ of acetone.
\end{abstract}

Key words: screen-printed PZT cantilevers, active carbon, volatile organic compounds sensing, electrospinning, nanofibers.

\section{Introduction}

The detection of volatile organic compounds (VOCs) at small concentrations is very important and interesting in different fields such as human health, industrial processes control or environmental monitoring [1]. Additionally, a good detector should comprise several aspects like reliability, coverage of the specific sensitivity range, low power consumption, and reduced cost among others [2]. In particular, cantilever based sensors have shown good characteristics for gas sensing at room temperature [3-4]. To perform detection of chemical compounds, specific sensitive layer is prior deposited on the cantilever to trap the different species. This phenomenon, which affects the mass of cantilever, induces both bending rigidity and surface stress changes. These mechanical modifications find expression in static or in dynamic modes respectively by curvature or resonance alterations [5]. More specifically, the addition of a high surface area absorbing sensitive overlayer onto the cantilevers can lead to unique opportunities for the fabrication of cantilever sensing devices and the detection of VOCs.

Particularly, in this work, we study the fabrication of active-carbon nanofiber-based sensitive layers onto gold coated screen-printed PZT cantilevers for the detection of VOCs.

\section{Experimental}

The self-actuating piezoelectric microcantilever of dimension $8 \times 2 \times 0.08 \mathrm{~mm}^{3}$ is obtained through screen-printing deposition technique onto a sacrificial layer [6]. It is basically composed of a PZT layer between two gold electrodes.

Active carbon with high surface area (1779 $\left.\mathrm{m}^{2} / \mathrm{g}\right)$ and small pore width $(1 \mathrm{~nm})$ was selected for its high adsorption capacity of volatile organic compounds. Electrospinning technique was chosen in order to produce high surface contact nanofiber-based sensitive layers onto the gold coated PZT cantilevers [7]. The electrospinning process was carried out using the setup shown in Fig. 1 with the following parameters: $10 \mu \mathrm{L} / \mathrm{min}$ solution feed rate, 12 $\mathrm{kV}$ voltage and $10 \mathrm{~cm}$ tip to collector distance. Active carbon powder (200 mg), prepared in our laboratory, was added to the polymer solution $(4 \mathrm{~g})$, composed by PVDF $15 \mathrm{wt} \%$ in dimethylformamide (DMF), and stirred for $3 \mathrm{~h}$.

The functionalized cantilevers were tested in a closed chamber at room conditions $\left(25^{\circ} \mathrm{C}\right.$ and $30 \% \mathrm{RH}$ ) and with different concentrations of VOCs. The impedance of the functionalized cantilevers was monitored during the experiments using an impedance meter (Wayne $\begin{array}{llll}\text { Kerr } & \text { Inc. } & 6530 & \end{array}$ 


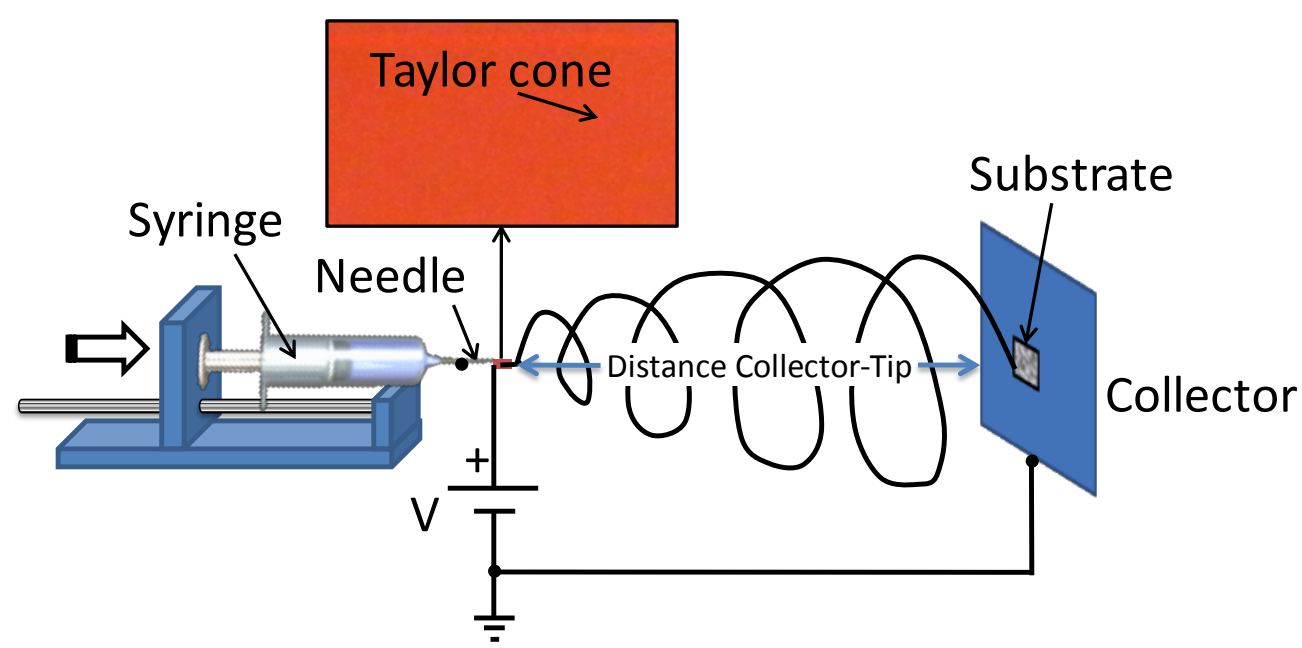

Fig. 1: Schematic representation of the setup used for the electrospinning deposition process and image of the Taylor cone formed during the deposition.

\section{Results and Discussion}

The fabricated active carbon nanofiber-based array can be observed in the SEM image of Fig. 2 , which expose a high surface interaction area.

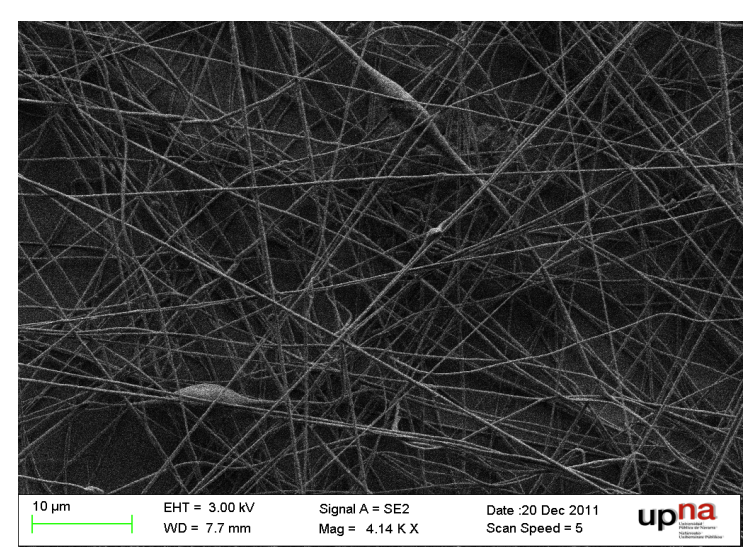

Fig. 2: SEM image of the nanofibers fabricated onto the cantilever.
The fiber average diameter is around $500 \mathrm{~nm}$. Electrical measurements of the fabricated samples confirmed the piezoelectric behavior of cantilevers and the presence of resonance modes after the active carbon nanofiber-based coating deposition. In particular, resonances can be observed in Fig. 3 at different frequencies such as $171.85 \mathrm{kHz}, 802.26 \mathrm{kHz}$ and $8.16 \mathrm{MHz}$.

Then, the device was introduced into a closed chamber and exposed to acetone at a concentration of $750 \mathrm{ppm}$ obtaining a shift of $971 \mathrm{~Hz}$ of the resonance frequency located at $802.26 \mathrm{kHz}$. The resonance shift of the device when it is exposed to acetone is represented in Fig. 4. The response time at room temperature is in the order of a few minutes, which shows promising results for the detection of different chemical species. Studies on the response time and selectivity of the sensors with different VOCs and other resonances are in progress.

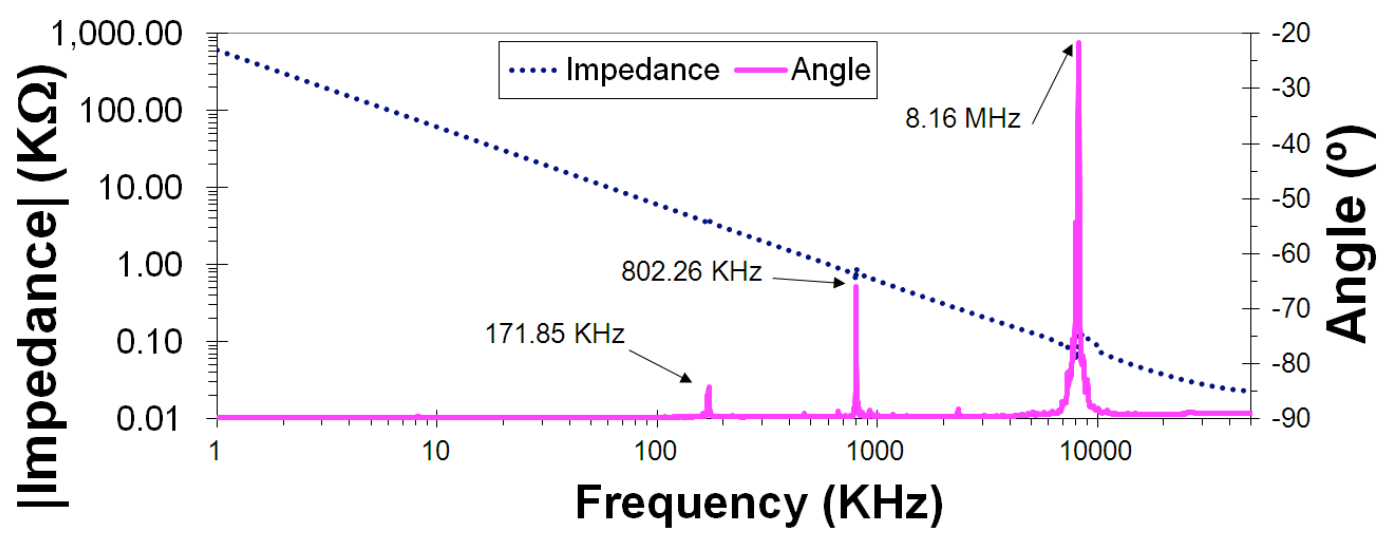

Fig. 3: Resonance frequencies of the cantilever after the nanofiber fabrication. 


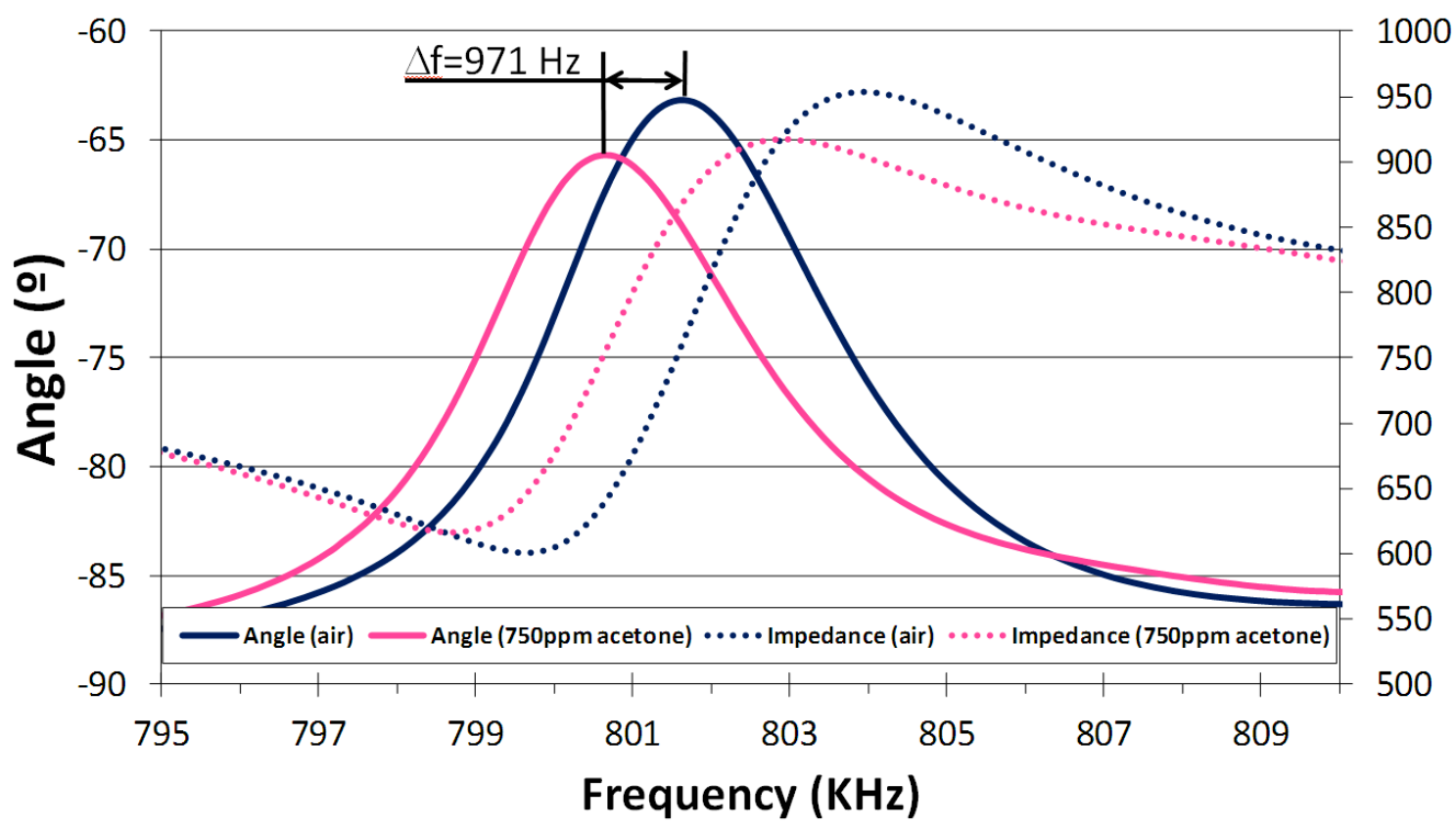

Fig. 4: Resonance frequency shift when the nanofiber coated cantilever is exposed to acetone in a concentration of $750 \mathrm{ppm}$ at $30 \% \mathrm{RH}$ and $25^{\circ} \mathrm{C}$.

\section{Conclusions}

Active-carbon nanofibers have been fabricated successfully onto gold coated screen-printed PZT cantilevers by means of the electrospinning deposition technique. The cantilevers revealed a resonance shift when they were exposed to acetone. This study shows promising results for the detection of different chemical species. A more profound study on the response time, selectivity and cross-sensitivity of the sensors with different VOCs, temperature and relative humidity as well as the sensitivities of the other resonances is still to be performed.

\section{Acknowledgements}

This work was funded in part by the Spanish Ministry of Education and Science-FEDER TEC2010-17805/MIC and Government of Navarre research grants.

\section{References}

[1] D. Lange, C. Hagleitner, A. Hierlemann, O. Brand, H. Baltes, Complementary Metal Oxide Semiconductor Cantilever Arrays on a Single Chip: Mass-Sensitive Detection of Volatile Organic Compounds, Anal. Chem. 74, 3084-3095 (2002) doi: 10.1021/ac011269j

[2] Y. Dong, W. Gao, Q. Zhou, Y. Zheng, Z. You, Characterization of the gas sensors based on polymer-coated resonant microcantilevers for the detection of volatile organic compounds, Analytica Chimica Acta 671 85-91 (2010); doi: 10.1016/j.aca.2010.05.007

[3] KM. Goeders, JS. Colton, LA. Bottomley, Microcantilevers: Sensing Chemical Interactions via Mechanical Motion, Chem. Rev. 108, 522-542 (2008); doi: 10.1021/cr0681041

[4] I. Dufour, F. Josse, SM. Heinrich, C. Lucat, C. Ayela, F. Ménil, O. Brand, Unconventional uses of microcantilevers as chemical sensors in gas and liquid media, Sensors and Actuators $B:$ Chem. in press (2011); doi: 10.1016/j.snb.2011.02.050

[5] T. Kanda, T. Morita, M.K. Kurosawa, T. Higuchi, A flat type touch probe sensor using PZT thin film vibrator, Sensors and Actuators A: Physical 83, 67-75 (2000); doi: 10.1016/S09244247(00)00298-3

[6] C. Lucat, P. Ginet, C. Castille, H. Debéda, F. Ménil, Microsystems elements based on freestanding thick-films made with a new sacrificial layer process, Microelectronics Reliability, 48(6), 872-875 (2008); 10.1016/j.microrel.2008.03.016

[7] JS. Im, SC. Kang, SH. Lee, YS. Lee, Improved gas sensing of electrospun carbon fibers based on pore structure, conductivity and surface modification, Carbon 48, 2573-2581 (2010); doi:10.1016/j.carbon.2010.03.045 